\title{
Impact of infection control training for interns on PICU-acquired bloodstream infections in a middle-income country
}

\author{
Yun Yun $\underline{\mathrm{Ng}}^{1,2}$, MD, MPaed, Mohamed El-Amin Abdel-Latif ${ }^{3,4}$, MRCPCH, FRACP, Chin Seng Gan ${ }^{1,5}$, MBBS, MPaed,
} Anis $\underline{\text { Siham }}^{5,6}$, MBBS, MPaed, Hasimah $\underline{\text { Zainol }}^{5}$, CCN, BNSc, Lucy Chai See $\underline{\operatorname{Lum}}^{1,5}$, MBBS, MRCP

\begin{abstract}
INTRODUCTION The present study aimed to determine the impact of an extended infection control training programme, which was conducted for all interns posted to the Department of Paediatrics, on the incidence of paediatric intensive care unit (PICU)-acquired bloodstream infections (BSIs) in University Malaya Medical Centre, Malaysia.

METHODS The development of nosocomial BSIs during the baseline period (1 January-31 October 2008) and intervention period (1 November-31 December 2009) was monitored. During the intervention period, all paediatric interns underwent training in hand hygiene and aseptic techniques for accessing vascular catheters.

RESULTS A total of 25 patients had PICU-acquired BSIs during the baseline period, while 18 patients had PICU-acquired BSIs during the intervention period (i.e. infection rate of 88 per 1,000 and 41 per 1,000 admissions, respectively). The infections were related to central venous catheters (CVCs) in 22 of the 25 patients who had PICU-acquired BSIs during the baseline period and 11 of the 18 patients who had PICU-acquired BSIs during the intervention period. Thus, the incidence rates of catheter-related BSIs were 25.2 per 1,000 CVC-days and 9.3 per 1,000 CVC-days, respectively ( $<<0.05)$. The Paediatric Risk of Standardised Mortality III score was an independent risk factor for PICU-acquired BSIs and the intervention significantly reduced this risk.

CONCLUSION The education of medical interns on infection control, a relatively low-cost intervention, resulted in a substantial reduction in the incidence of PICU-acquired BSIs.
\end{abstract}

Keywords: extended infection control training programme, interns, nosocomial bloodstream infection, paediatric intensive care unit

\section{INTRODUCTION}

Healthcare-associated infections (HCAIs), particularly bloodstream infections (BSIs) acquired in the intensive care unit (ICU), pose a serious risk to the critically ill. Such infections are associated with prolonged length of stay, ${ }^{(1-3)}$ higher treatment costs, ${ }^{(4,5)}$ and increased morbidity and mortality. ${ }^{(1,2,6)}$ Central line-associated BSIs (CLABSIs) have been demonstrated to be preventable through the implementation of multidimensional strategies, including multifaceted education, hand hygiene, cleaning skin with chlorhexidine, maximal barrier precautions for central venous catheter (CVC) insertion, redesigning physical barriers between patients' beds and the removal of unnecessary catheters. ${ }^{(7-11)}$ Proper CVC insertion and maintenance play a vital role in preventing infections, particularly CLABSIs. ${ }^{(1-13)}$ The rate of CLABSIs in the paediatric population has dropped markedly in developed countries due to significant investment in the aforementioned multidimensional strategies. ${ }^{(7-11)}$ According to the National Healthcare Safety Network's 2012 report, there was a pooled rate of 1.4 laboratory-Confirmed CLABSIs per 1,000 CVC-days in paediatric medical ICUs in the United States. ${ }^{(14)}$ The implementation of such multidimensional strategies for infection control requires institutional leadership and commitment to providing resources, teamwork, collaboration and expertise, in order to be effective and sustainable. ${ }^{(12,15,16)}$
Intensive care, particularly paediatric intensive care, is a fledgling specialty in resource-limited countries. Despite the major potential impact of any failure in infection control, it receives little attention compared to other priorities. Although HCAI surveillance systems are in place in many developed countries at the national/subnational level, only 23 of 147 (15.6\%) developing countries reported a functioning $\mathrm{HCAl}$ national surveillance system in a survey conducted by the World Health Organization. ${ }^{(17)}$ A systematic review and meta-analysis revealed a striking difference between the incidence of $\mathrm{ICU}$-acquired infections in developing countries (a pooled density of 47.9 per 1,000 patient-days) and that in the United States (an estimated 13.6 per 1,000 patientdays). ${ }^{(18,19)}$ The lack of a comprehensive surveillance system for HCAls in resource-limited countries puts critically ill patients at risk. A review of the incidence of CLABSIs in resource-limited countries revealed the stark reality of poor infection control overcrowded ICUs, insufficient rooms for isolation, lack of sinks, lack of alcohol hand rubs and chlorhexidine, and the use of vented intravenous containers. ${ }^{(18,20)}$ Unless the problem of basic infection control practices is addressed, it is unlikely that implementing the aforementioned multidimensional strategies would be sufficient to prevent CLABSIs in the hospitals of these countries. ${ }^{(20)}$

Nosocomial infection surveillance initiated by the paediatric ICU (PICU) at University Malaya Medical Centre, Malaysia, and

${ }^{1}$ Department of Paediatrics, Faculty of Medicine, University Malaya, ${ }^{2}$ Institute Paediatrics, Hospital Kuala Lumpur, Malaysia, ${ }^{3}$ School of Clinical Medicine, Australian National University, ${ }^{4}$ Department of Neonatology, Centenary Hospital for Women and Children, Canberra Hospital, Australia, ${ }^{5}$ Paediatric Intensive Care Unit, University Malaya Medical Center, ${ }^{6}$ Department of Paediatrics, Faculty of Medicine, Universiti Teknologi MARA, Malaysia

Correspondence: Prof Lucy CS Lum, Professor, Department of Paediatrics, Faculty of Medicine, University Malaya, 50603 Kuala Lumpur, Malaysia. lumcs@ummc.edu.my 
Table I. Timeline of the educational training programme.

\begin{tabular}{|c|c|}
\hline Date of implementation & Item(s) covered \\
\hline January 2003 & $\begin{array}{l}\text { - Use of single-use sterile alcohol wipes instead of cotton balls impregnated with antiseptic } \\
\text { - Prohibiting the reuse of single-use vials } \\
\text { - Disposal of fluids used for dilution of parenteral medications that were more than } 24 \text { hours old }\end{array}$ \\
\hline January 2004 & - Mandatory nurse education on hand hygiene with chlorhexidine wash* \\
\hline January 2005 & $\begin{array}{l}\text { Mandatory nurse and doctor education on: } \\
\text { - Use of sterile gloves before central venous catheter (CVC) insertion or manipulation } \\
\text { - Use of sterile gauze or transparent sterile dressing to cover the insertion site } \\
\text { - Maintenance of sterile dressing (i.e. changing gauze every } 48 \text { hours and transparent dressings } \\
\text { every seven days, and removing CVCs when not needed) } \\
\text { - Use of closed intravenous systems (changing the administration set every } 96 \text { hours unless it } \\
\text { was used for nutrition or blood products, in which case it should be changed every } 24 \text { hours) }\end{array}$ \\
\hline January 2007 & - Use of CVC insertion kits and maximal sterile barrier precautions during CVC insertion \\
\hline January 2008 & $\begin{array}{l}\text { - Training of PICU doctors on the disinfection of line hubs, needleless connectors and injection } \\
\text { ports before CVC assessment } \\
\text { - Real-time feedback about CLABSI control intervention }\end{array}$ \\
\hline November 2008 & - Training of all paediatric interns (i.e. start of the intervention period) \\
\hline
\end{tabular}

*Povidone-iodine used for skin preparation as chlorhexidine was not available. CLABSIs: central line-associated bloodstream infections; PICU: paediatric intensive care unit

conducted in 2001 revealed a BSI rate of 243 per 1,000 patients in the PICU. (21) This unacceptably high incidence prompted a definitive approach to infection control; an educational training programme targeted at all PICU nursing staff, residents and interns who were rotated to the PICU was introduced in 2002. To assess the impact of infection control training for interns, the training was extended to all paediatric interns (including those who were not rotated to the PICU but who would provide coverage outside of working hours) from 1 November 2008. We hypothesised that the education of these paediatric interns would positively impact the rate of nosocomial BSIs in the PICU.

\section{METHODS}

This study was performed in the PICU of University Malaya Medical Centre in Kuala Lumpur, Malaysia. It was approved by the ethics committee of the medical centre, which waived the requirement for written informed consent. The 10-bed tertiary care multidisciplinary $\mathrm{PICU}$, which is under the administration of the Department of Paediatrics, admits approximately 400 critically ill patients per year. During working hours, clinical management was undertaken by the PICU team, which comprised a paediatric intensivist, two specialists, four residents and two interns who were placed in the PICU full-time for the duration of their two-month rotation. After working hours, due to staff shortage, PICU coverage was provided by medical staff and interns who were not part of the PICU team and had not been trained in infection control. The nurse to patient ratio averaged 1:1.5 and varied between $1: 1$ and $1: 2$, according to patient acuity. An educational training programme to reduce CLABSIs in the PICU was introduced to PICU physicians and nurses in several stages between 2002 and the start of the study in 2008 (Table I).

Patients admitted to the PICU between 1 January 2008 and 31 December 2009 were enrolled into the study. The baseline period was between 1 January and 31 October 2008, while the intervention period was between 1 November 2008 and 31 December 2009. During the intervention period, all paediatric interns, regardless of whether or not they were posted to PICU, underwent training to practice hand hygiene and aseptic technique for accessing CVCs, peripheral venous cannulas and arterial catheters at the start of their rotation in the Department of Paediatrics (see Appendix). Training was provided by the paediatric intensivist and specialist. The training sessions involved groups of 2-4 interns, lasted for two hours and were followed by the evaluation of each intern's performance in infection control practices. Training and evaluation were repeated until the interns achieved the required standard.

Patients were excluded if they were in an unstable haemodynamic state that required continuous cardiopulmonary resuscitation at the time of admission. Patients included in the study were monitored daily by a trained nurse for invasive device utilisation and the development of nosocomial BSIs (from the time of admission to up to 48 hours after PICU discharge). The primary outcome measure was the development of a PICU-acquired BSI, which included clinical sepsis and laboratory-confirmed BSI, and CLABSIs per 1,000 CVC-days. Primary BSIs included both clinical sepsis and laboratory-confirmed BSI with a positive blood culture not related to infection at another site; (22) ICU-associated infection was defined as an infection that was not present or incubating during the patient's admission to the ICU, but became apparent during the ICU stay or within 48 hours after transfer from the ICU. ${ }^{(23,24)}$ A BSI was considered to have been acquired in the PICU if the positive blood culture was obtained more than 48 hours after the patient's admission to the unit. Clinical sepsis was diagnosed if the patient had at least one of the following: a fever, hypotension or oliguria; a physician-instituted treatment for sepsis with no apparent infection at another site; or a negative blood culture or no blood culture performed. ${ }^{(22,25)}$ CLABSI was defined as a primary BSI if the patient had a central line within the 48-hour period before the development of the BSI and it was not related to an infection at another site. ${ }^{(26)}$ Infection incidence was expressed as a percentage of the total number of patients with PICU-acquired BSIs divided by the total number of patients enrolled during the respective period. ${ }^{(24)}$ 
All statistical analyses were performed using IBM SPSS Statistics version 20.0 (IBM Corp, Armonk, NY, USA). Data was presented as number (percentage) or median (interquartile range). For actuarial analysis, the event of interest was 'nosocomial BSI' and the time-to-event was the 'number of nosocomial BSI-free days'. Patients with no events were considered as right censored observations. The Kaplan-Meier product-limit method ${ }^{(27)}$ was used to estimate the univariate differences in the actuarial analysis, with differences compared using the two-sided log rank test. ${ }^{(28)}$ Hazard ratios were calculated using the multiple Cox proportional hazards regression model, ${ }^{(29,30)}$ after adjusting for significant and clinically important baseline characteristics. In this model, the criterion for entry and removal were $p<0.05$ and $p>0.10$, respectively. The assumption of proportionality was confirmed by plotting the log negative log of the Kaplan-Meier estimates of the survival function versus the log of time, which resulted in parallel curves. All analyses were pre-specified. The level of statistical significance for all analyses was set at $p<0.05$, using two-tailed comparisons.

\section{RESULTS}

A total of 110 interns were posted to the Department of Paediatrics during the study period (1 January 200831 December 2009): 45 were posted during the baseline period, while 65 were posted during the intervention period (Fig. 1). During the baseline period, only the 13 interns who were rotated to the PICU were trained in infection control. During the intervention period, all the 65 interns who were posted to the Department of Paediatrics underwent training in infection control. The number of interns who provided PICU coverage after working hours during the baseline and intervention periods were 41 and 61, respectively.

A total of 749 patients were admitted to the PICU between 1 January 2008 and 31 December 2009. Among these 749 patients, 28 patients were excluded as they were in an unstable haemodynamic state that required continuous cardiopulmonary resuscitation at the time of admission. Of the 721 remaining patients, 285 were enrolled during the baseline period and 436 were enrolled during the intervention period (Fig. 2). Table II shows the characteristics of the patients in the baseline and intervention groups. Patient characteristics, such as age, gender, reasons for PICU admission, Paediatric Risk of Standardised Mortality (PRISM) III score, inotrope administration and elective admissions during the baseline and intervention periods were similar. The rates of invasive device utilisation were similar between the baseline and intervention groups for mechanical ventilators and indwelling urinary catheters.

There was a significant decrease in the use of CVCs and arterial catheters during the intervention period (Fig. 3). The start of the intervention period (i.e. 1 November 2008) was accompanied by a simultaneous decrease in BSIs, which remained low throughout the intervention period. The decreased utilisation of CVCs and arterial catheters during May 2009 to August 2009 was due to an unforeseen shortage of paediatricsized CVCs and arterial catheters; the supply was re-established

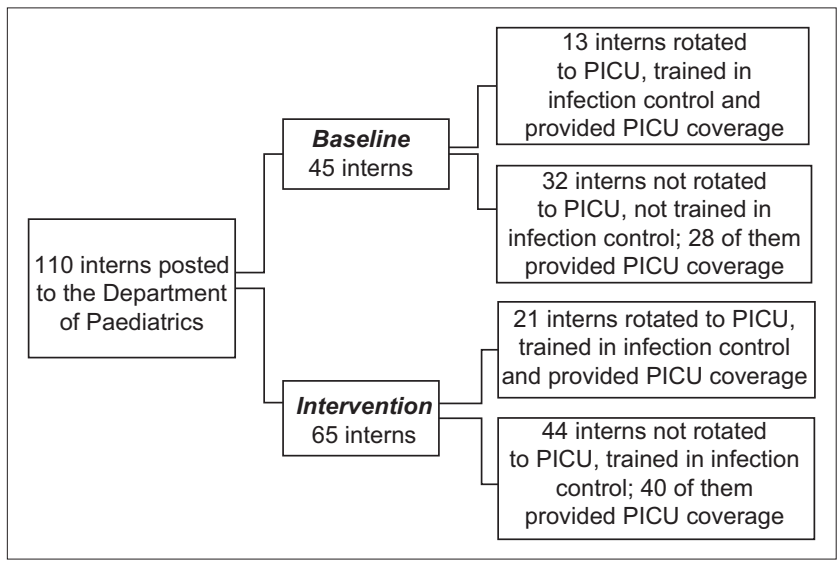

Fig. 1 Flow chart shows the distribution of interns to the paediatric intensive care unit (PICU) during the study period.

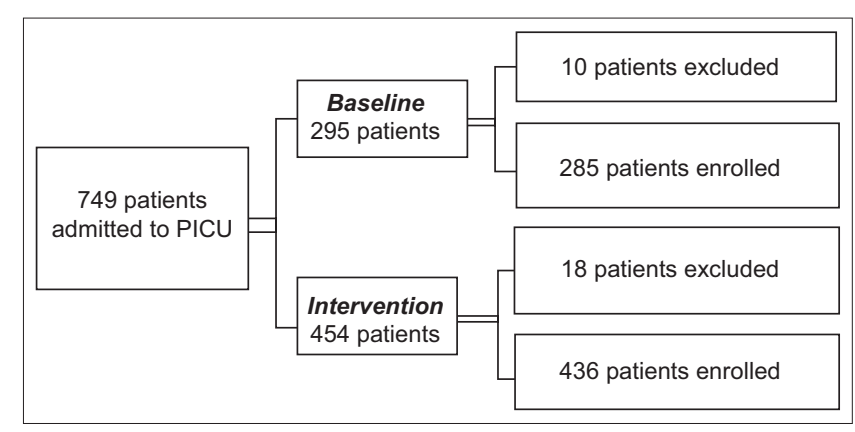

Fig. 2 Flow chart shows the distribution of paediatric intensive care unit (PICU) patients during the study period.

during the last four months of the intervention period. Despite the increased device utilisation from September 2009 to December 2009, the rate of BSIs was significantly lower during the intervention period than during the baseline period. Dayby-day Kaplan-Meier product-limit BSIs, stratified according to the baseline and intervention periods are shown in Fig. 4. The graphs were truncated at day 40 of admission as the number of BSIs remained mostly unchanged afterwards. The overall risk of BSI was higher during the baseline period as compared to the intervention period $(p=0.048)$.

The median number of CVC-days during the baseline and intervention periods was 874 days and 1,183 days, respectively. During the baseline and intervention periods, 25 patients and 18 patients had PICU-acquired BSIs, respectively. In other words, the incidence rates of PICU-acquired BSIs were 88 per 1,000 admissions and 41 per 1,000 admissions, respectively. Of the 25 patients with PICU-acquired BSIs during the baseline period, 22 cases were CVC-related, giving a CLABSI incidence rate of 25.2 per 1,000 CVC-days. Of the 18 patients with PICU-acquired BSIs during the intervention period, 11 cases were CVC-related, giving a CLABSI incidence rate of 9.3 per 1,000 CVC-days. This translates to a $63 \%$ reduction in CLABSI during the intervention period ( $p<0.05$ ). Multivariate analysis revealed that the PRISM III score, but not the durations of CVC or arterial catheter usage, was a significant risk factor for the development of PICU-acquired BSI (Table III). It also showed that the risk was significantly reduced during the intervention period. 
Table II. Characteristics of included patients admitted to the paediatric intensive care unit (PICU) before and during the intervention.

\begin{tabular}{|c|c|c|c|}
\hline \multirow[t]{2}{*}{ Characteristic } & \multicolumn{2}{|c|}{ No. (\%) } & \multirow[t]{2}{*}{ p-value } \\
\hline & Baseline $(n=285)$ & Intervention $(n=436)$ & \\
\hline Age* $^{*}(y r)$ & $1.0(0.2-5.6)$ & $0.8(0.1-4.9)$ & 0.219 \\
\hline Gender & & & 0.188 \\
\hline Male & $155(54.4)$ & $260(59.6)$ & \\
\hline Female & $130(45.6)$ & $176(40.4)$ & \\
\hline Inotrope use & 68 (23.9) & $97(22.2)$ & 0.680 \\
\hline Elective admission & $53(18.6)$ & $72(16.5)$ & 0.589 \\
\hline \multicolumn{4}{|l|}{ Reason for PICU admission } \\
\hline Post-surgery support & $82(28.8)$ & $112(25.7)$ & 0.406 \\
\hline Respiratory support & $102(35.8)$ & $165(37.8)$ & 0.642 \\
\hline Cardiovascular support & $33(11.6)$ & $64(14.7)$ & 0.280 \\
\hline Respiratory and haemodynamic support & $52(18.2)$ & $72(16.5)$ & 0.624 \\
\hline Neurological support & $16(5.6)$ & $23(5.3)$ & 0.995 \\
\hline \multicolumn{4}{|l|}{ Invasive device utilisation } \\
\hline Mechanical ventilation & $167(58.6)$ & $250(57.3)$ & 0.797 \\
\hline CVC & $98(34.4)$ & $116(26.6)$ & 0.031 \\
\hline Peripheral arterial catheter & $156(54.7)$ & $208(47.7)$ & 0.077 \\
\hline Indwelling urinary catheters & $131(46.0)$ & $180(41.3)$ & 0.245 \\
\hline \multicolumn{4}{|l|}{ Duration of invasive device utilisation* (day) } \\
\hline Mechanical ventilation & $4.0(2.0-10.0)$ & $5.0(2.0-8.0)$ & 0.202 \\
\hline CVC & $5.5(3.0-10.0)$ & $6.0(3.0-12.0)$ & 0.369 \\
\hline Peripheral arterial catheter & $5.0(3.0-10.0)$ & $4.0(3.0-7.0)$ & 0.041 \\
\hline Indwelling urinary catheters & $4.0(2.0-7.0)$ & $4.0(3.0-7.0)$ & 0.360 \\
\hline \multicolumn{4}{|l|}{ Type of BSI } \\
\hline Laboratory-confirmed & $13(4.6)$ & $10(2.3)$ & 0.140 \\
\hline Clinical sepsis & $12(4.2)$ & $8(1.8)$ & 0.095 \\
\hline Total BSI & $25(8.8)$ & $18(4.1)$ & 0.016 \\
\hline CLABSI & $22(7.7)$ & $11(2.5)$ & 0.002 \\
\hline Time to onset of BSI* (day) & $5.0(2.0-9.0)$ & $5.0(3.0-9.0)$ & 0.624 \\
\hline CVC-days & 874 & 1,183 & \\
\hline CLABSI per 1,000 CVC-days & 25.2 & 9.3 & $<0.001$ \\
\hline Length of PICU stay* (day) & $5.0(2.0-11.5)$ & $5.0(3.0-9.0)$ & 0.459 \\
\hline PRISM III score & $5.0(2.0-10.5)$ & $5.0(3.0-10.0)$ & 0.791 \\
\hline PICU mortality & $26(9.1)$ & $39(8.9)$ & 0.935 \\
\hline
\end{tabular}

*Data expressed as median (interquartile range). BSI: bloodstream infection; CLABSI: central line-associated bloodstream infection; CVC: central venous catheter; PRISM: Paediatric Risk of Standardised Mortality

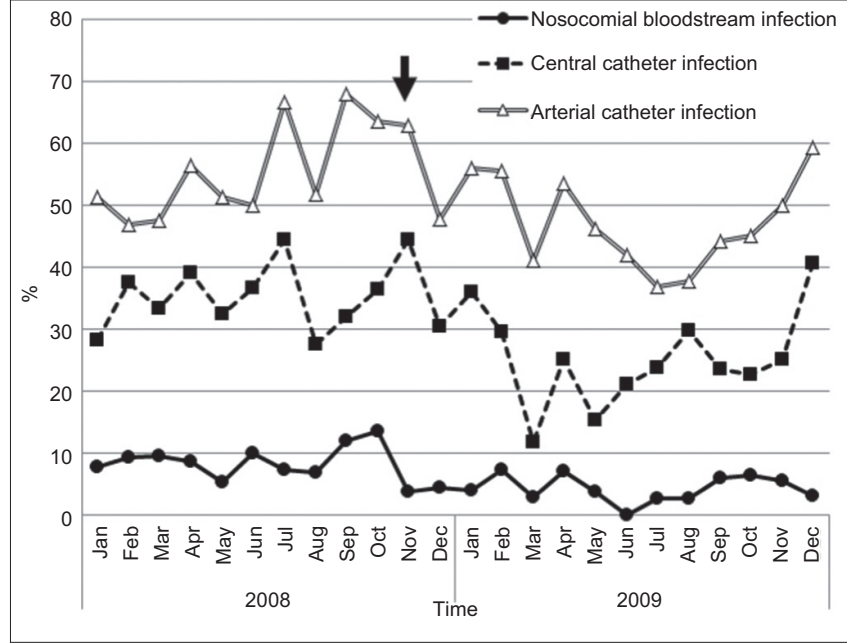

Fig. 3 Rates of bloodstream infection and use of central venous catheters and arterial catheters among patients admitted to the paediatric intensive care unit before and during the intervention. The arrow shows the start of the intervention period (i.e. 1 November 2008).

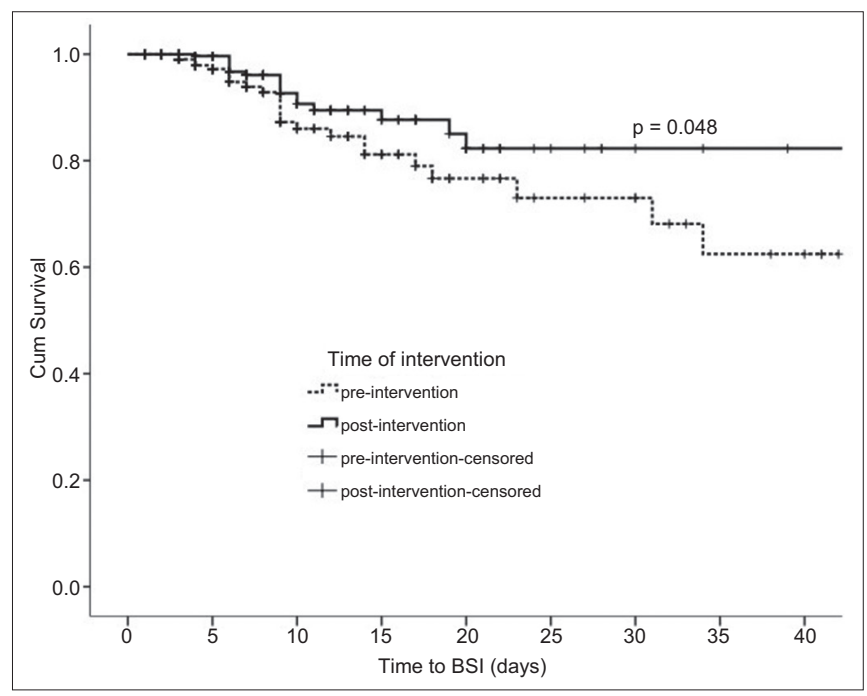

Fig. 4 Day-by-day Kaplan-Meier product-limit of bloodstream infections (BSIs) among patients admitted to the paediatric intensive care unit before and during the intervention. The graph was truncated at day 40 of admission. 
Table III. Multivariate Cox regression analysis of the factors associated with bloodstream infections among patients admitted to the paediatric intensive care unit before and during the intervention.

\begin{tabular}{lcc}
\hline Variable & Hazard ratio $(95 \% \mathrm{CI})$ & p-value \\
\hline $\begin{array}{l}\text { Intervention period vs. } \\
\text { baseline period }\end{array}$ & $0.443(0.199-0.989)$ & 0.047 \\
$\begin{array}{l}\text { Duration of central } \\
\text { venous catheter use (day) }\end{array}$ & $1.014(0.991-1.037)$ & 0.243 \\
$\begin{array}{l}\text { Duration of arterial } \\
\text { catheter use (day) }\end{array}$ & $1.018(0.979-1.058)$ & 0.377 \\
PRISM III score & $1.079(1.016-1.145)$ & 0.013 \\
\hline
\end{tabular}

All covariates that were included in the multivariate models are included in the table. $\mathrm{Cl}$ : confidence interval; PRISM: Paediatric Risk of Standardised Mortality

\section{DISCUSSION}

During the baseline period, less than $30 \%$ of all the interns who provided after-working-hours coverage in the PICU received training in infection control. The extension of this training to all interns in the Department of Paediatrics (i.e. during the intervention period) was associated with reduced rates of both laboratory-confirmed BSI and clinical sepsis, by $28 \%$ in total BSI and $63 \%$ in CLABSI per 1,000 CVC-days. Patient characteristics, such as age, gender, severity of illness, reason for admission, and use of inotropes, invasive mechanical ventilation and continuous indwelling urinary catheters, were similar during the baseline period and the intervention period. Although the decreased use of CVCs and arterial catheters during the intervention period may have impacted the BSI rates, multivariate analysis revealed that the use of these devices was not a significant risk factor for the development of PICU-acquired BSIs. Furthermore, the increased use of these devices during the last four months of the intervention period did not result in increased BSI rates.

To the best of our knowledge, the present study is the first to report on how the training of all interns in infection control practices affects the incidence of nosocomial BSIs in the PICU of a middle-income country. While medical interns who were rotated to the PICU performed tasks that involve patient contact and central vascular access, it was reported that only $64 \%$ of them had a sufficient level of awareness about infection control practices. ${ }^{(31)}$ The education and training of healthcare providers who insert and maintain catheters, which is a key intervention in the prevention of CLABSIs, often does not involve interns, even though this could have a potentially dramatic impact on the rate of infections in the PICU. ${ }^{(31-33)}$

The PICU in the present study is typical of those found in middle-income countries. Dependence on itinerant staff after working hours makes infection control, as well as its education and surveillance, challenging. This is especially the case when infection control practices receive little attention in the other areas of the hospital. Due to limited resources, process surveillance and feedback on the performance of infection control practices were not performed in the present study. Sustained compliance to hand hygiene, which is fundamental to the control of nosocomial infections, is poor among healthcare workers. ${ }^{(34)}$ The implementation of frequent performance feedback, in addition to education and training (as recommended by the International
Nosocomial Infection Control Consortium), has been shown to increase hand hygiene adherence from $23.1 \%$ to $64.5 \%$ in two ICUs. In that study, the overall reduction of nosocomial infections was from 47.55 per 1000 patient-days to 27.93 per 1000 patientdays. ${ }^{(32)}$ Compliance with CVC-site care has been shown to improve with sequential implementation of an education and performance feedback programme; the implementation of an educational programme was also shown to reduce the rate of intravenous device-associated BSIs, with further reductions noted after a performance feedback programme was implemented. ${ }^{(35)}$

The present study was not without limitations. As it was conducted in a single PICU in a teaching hospital, the results cannot be considered to be representative of all PICUs from middle-income countries. The unintentional decreased utilisation of CVCs and arterial catheters during part of the intervention period may also have affected the findings on the impact of the intervention programme. Although the rate of BSIs was reduced after the implementation of the intervention programme, it was still higher than that reported in the United States and other developing countries that have implemented CVC bundle care. ${ }^{(36)}$

In conclusion, our study showed that the relatively low-cost investment of educating interns in infection control has the potential to substantially reduce the incidence of nosocomial BSIs in PICUs. We believe that the implementation of similar strategies in other parts of the hospital can lead to a significant reduction in the incidence of nosocomial BSIs in vulnerable patients.

\section{ACKNOWLEDGEMENTS}

We extend our appreciation to $\mathrm{MM} \mathrm{Ho}$, research nurse, and Dr A Kassim, postgraduate Master's student in paediatrics, for initiating the first surveillance on nosocomial infections in University Malaya Medical Centre's PICU in 2001, and F Bakar, ward manager, for the moral and technical support given. We also thank the staff of the PICU at University Malaya Medical Centre for their cooperation and assistance in the study. This study was funded by the University Malaya Research Grant (RG277/10HTM) and the Short Term Research Grant (PJP) University of Malaya (FS250-2008C), and received support from the Ministry of Science, Technology and Innovation, Malaysia (060101SF0512 and ERGS/1/2011).

\section{REFERENCES}

1. Pittet D, Harbarth $S$, Ruef $C$, et al. Prevalence and risk factors for nosocomial infections in four university hospitals in Switzerland. Infect Control Hosp Epidemiol 1999; 20:37-42.

2. Rosenthal VD, Guzman S, Orellano PW. Nosocomial infections in medicalsurgical intensive care units in Argentina: attributable mortality and length of stay. Am J Infect Control 2003; 31:291-5.

3. Barnett AG, Graves N, Rosenthal VD, Salomao R, Rangel-Frausto MS. Excess length of stay due to central line-associated bloodstream infection in intensive care units in Argentina, Brazil, and Mexico. Infect Control Hosp Epidemiol 2010; 31:1106-14.

4. Slonim AD, Kurtines HC, Sprague BM, Singh N. The costs associated with nosocomial bloodstream infections in the pediatric intensive care unit. Pediatr Crit Care Med 2001; 2:170-4.

5. Elward AM, Hollenbeak CS, Warren DK, Fraser VJ. Attributable cost of nosocomial primary bloodstream infection in pediatric intensive care unit patients. Pediatrics 2005; 115:868-72.

6. Richards MJ, Edwards JR, Culver DH, Gaynes RP. Nosocomial infections 
in pediatric intensive care units in the United States. National Nosocomia Infections Surveillance System. Pediatrics 1999; 103:e39.

7. Bhutta A, Gillian C, Honeycutt M, et al. Reduction of bloodstream infections associated with catheters in paediatric intensive care unit: stepwise approach. BMJ 2007; 334:362-5.

8. Warren DK, Zack JE, Mayfield JL, et al. The effect of an education program on the incidence of central venous catheter-associated infection in a medical ICU. Chest 2004; 126:1612-8.

9. Sherertz RJ, Ely EW, Westbrook DM, et al. Education of physicians-intraining can decrease the risk for vascular catheter infection. Ann Intern Med 2000; 132:641-8.

10. Eggimann $\mathrm{P}$, Harbarth S, Constantin $\mathrm{MN}$, et al. Impact of a prevention strategy targeted at vascular-access care on incidence of infections acquired in intensive care. Lancet 2000; 355:1864-8.

11. Provonost $P$, Needham $D$, Berenholtz $S$, et al. An intervention to decrease catheter-related bloodstream infections in the ICU. N Engl J Med 2006 355:2725-32.

12. Costello JM, Morrow DF, Graham DA, et al. Systematic intervention to reduce central line-associated bloodstream infection rates in a pediatric cardiac intensive care unit. Pediatrics 2008; 121:915-23.

13. Yogaraj JS, Elward AM, Fraser VJ. Rate, risk factors, and outcomes of nosocomial primary bloodstream infection in pediatric intensive care unit patients. Pediatrics 2002; 110:481-5.

14. Dudeck MA, Weiner LM, Allen-Bridson K, et al. National Healthcare Safety Network (NHSN) report, data summary for 2012, Device-associated module. Am J Infect Control 2013; 41:1148-66.

15. Miller MR, Griswold M, Harris JM 2nd, et al. Decreasing PICU catheterassociated bloodstream infections: NACHRI's quality transformation efforts Pediatrics 2010; 125:206-13.

16. Wheeler DS, Giaccone MJ, Hutchinson N, et al. A hospital-wide qualityimprovement collaborative to reduce catheter-associated bloodstream infections. Pediatrics 2011; 128:e995-e1004.

17. World Health Organization. The Burden of Health Care-Associated Infection Worldwide. A Summary. Available at: http://www.who.int/gpsc/ country_work/summary_20100430_en.pdf. Accessed March 23, 2014.

18. Allegranzi B, Bagheri Nejad S, Combescure C, et al. Burden of endemic health-care-associated infection in developing countries: systematic review and meta-analysis. Lancet 2011; 377:228-41.

19. Klevens RM, Edwards JR, Richards CL Jr, et al. Estimating health careassociated infections and deaths in U.S. hospitals, 2002. Public Health Rep 2007; 122:160-6.

20. Rosenthal VD. Central line-associated bloodstream infections in limitedresource countries: a review of the literature. Clin Infect Dis 2009; 49:1899-907.

21. Kassim A. Nosocomial infection in paediatric intensive care unit (PICU),
University Malaya Medical Centre, Kuala Lumpur (dissertation). Kuala Lumpur: University Malaya, 2002.

22. Hugonnet S, Sax H, Eggimann P, Chevrolet JC, Pittet D. Nosocomial bloodstream infection and clinical sepsis. Emerg Infect Dis 2004; 10:76-81.

23. Horan TC, Andrus M, Dudeck MA. CDC/NHSN surveillance definition of health care-associated infection and criteria for specific types of infections in the acute care setting. Am J Infect Control 2008; 36:309-32.

24. Garner JS, Jarvis WR, Emori TG, Horan TC, Hughes JM. CDC definition for nosocomial infection. In: Olmsted RN, eds. Infection control and applied epidemiology: principles and practice. St Louis: Mosby, 1996: A1-20.

25. Horan TC, Gaynes RP. Surveillance of nosocomial infections. In: Mayhall CG, eds. Hospital Epidemiology and Infection Control. 3rd ed. Philadelphia: Lippincott Williams \& Wilkins, 2004: 1659-702.

26. O'Grady NP, Alexander M, Burns LA, et al; Healthcare Infection Control Practices Advisory Committee (HICPAC). Guidelines for the prevention of intravascular catheter-related infections. Clin Infect Dis 2011; 52:e162-93.

27. Kaplan EL, Meier P. Nonparametric estimation from incomplete observations. J Am Stat Assoc 1958; 53:457-81

28. Mantel N. Evaluation of survival data and two new rank order statistics arising in its consideration. Cancer Chemother Rep 1966; 50:163-70.

29. Cox DR. Regression models and life-tables. J R Stat Soc 1972; B34:187-220.

30. Hosmer DW, Lemeshow S. Applied logistic regression. New York: Wiley, 1989.

31. Al-Zahrani S, Al-Amry F, Ghonaim M, Abo-Salem O. Awareness \& knowledge of medical students and interns about infection control measures. Int J Med Sci Public Health 2013; 2:317-23.

32. Sherertz RJ, Ely EW, Westbrook DM, et al. Education of physicians-in training can decrease the risk for vascular catheter infection. Ann Intern Med 2000; 132:641-8

33. Iqbal Y. Residents help raise the bar on infection control efforts. In: American College of Physicians [online]. Available at: http://www. acpinternist.org/archives/2004/10/infection.htm. Accessed October 6, 2013.

34. Rosenthal VD, Guzman S, Safdar N. Reduction in nosocomial infection with improved hand hygiene in intensive care units of a tertiary care hospital in Argentina. Am J Infect Control 2005; 33:392-7.

35. Rosenthal VD, Guzman S, Pezzotto SM, Crnich Cl. Effect of an infection control program using education and performance feedback on rates of intravascular device-associated bloodstream infections in intensive care units in Argentina. Am J Infect Control 2003; 31:405-9.

36. Rosenthal VD, Ramachandran B, Villamil-Gómez W, et al. Impact of a multidimensional infection control strategy on central line-associated bloodstream infection rates in pediatric intensive care units of five developing countries: findings of the International Nosocomial Infection Control Consortium (INICC). Infection 2012; 40:415-23. 


\section{APPENDIX}

\section{PRELIMINARY ORIENTATION OF HOUSE OFFICERS POSTED TO DEPARTMENT OF PAEDIATRICS}

Date:

Name of House Officer:

Date of Posting to Department of Paediatrics:

Please note that you have to present yourself to the PICU within 48 hours of being posted to the Department of Paediatrics

\section{Orientation of PICU by Clinical Nurse Consultant \& PICU lecturer/specialist}

\begin{tabular}{|c|c|c|c|}
\hline \multicolumn{2}{|c|}{ Procedure } & \multirow{2}{*}{$\begin{array}{l}\text { Demonstrated by specialist or } \\
\text { senior MO (signature } \& \text { date) }\end{array}$} & \multirow{2}{*}{$\begin{array}{l}\text { Assessed by } \\
\text { Sister __ (date) } \\
\text { Satisfactory } \\
\square \text { To repeat }\end{array}$} \\
\hline 1. & Hand-washing & & \\
\hline 2. & Access of arterial lines & & $\begin{array}{l}\square \text { Satisfactory } \\
\square \text { To repeat }\end{array}$ \\
\hline 3. & Access of central venous lines and administration of medications & & $\begin{array}{l}\square \text { Satisfactory } \\
\square \text { To repeat }\end{array}$ \\
\hline 4. & Access of peripheral venous line and administration of medications & & $\begin{array}{l}\square \text { Satisfactory } \\
\square \text { To repeat }\end{array}$ \\
\hline 5. & Arterial blood gas analyser & & $\begin{array}{l}\square \text { Satisfactory } \\
\square \text { To repeat }\end{array}$ \\
\hline 6. & Capillary gas sampling & & $\begin{array}{l}\square \text { Satisfactory } \\
\square \text { To repeat }\end{array}$ \\
\hline 7. & Safety in blood transfusion & & $\begin{array}{l}\square \text { Satisfactory } \\
\square \text { To repeat }\end{array}$ \\
\hline 8. & Safety in medication administration & & $\begin{array}{l}\square \text { Satisfactory } \\
\square \text { To repeat }\end{array}$ \\
\hline
\end{tabular}

- Important points to note during procedures:

- Preparation before access of vascular devices

- Inform patient or parent before procedure

- Perform the actual procedure with

- Aseptic considerations and neatness

- Flushing the line to maintain patency

- Ensuring no air bubbles in the vascular system

- If your assessment is not satisfactory in any of these areas, you will be assessed at another date before your first call in the PICU.

Once the assessment has been completed satisfactorily, please submit this document to PICU Ward Manager. 